\title{
THE GENERATION OF NONLINEAR EQUIVARIANT DIFFERENTIAL OPERATORS ${ }^{1}$
}

\author{
ROBERT DELVER
}

\begin{abstract}
Finite generation results are given for the set of smooth nonlinear differential operators: $C^{\infty}(M, N) \rightarrow C^{\infty}(M, \mathbf{R})$ of order $<k$ which are equivariant with respect to the action of a Lie group on the base manifold $\boldsymbol{M}$.
\end{abstract}

1. Introduction. Let $G$ be a Lie group acting by diffeomorphisms $\phi_{g}, g \in G$, on a smooth manifold $M, N$ a smooth manifold and let $\mathscr{D}_{k}, k \in$ $\{\infty, 1,2,3, \ldots\}$, denote the real vector space of smooth nonlinear differential operators of order $\leqslant k$ of $C^{\infty}(M, N)$ into $C^{\infty}(M, \mathbf{R})$. The action of $G$ on $M$ lifts to $C^{\infty}(M, N)$ by $g \cdot f=f \circ \phi_{g}^{-1}, f \in C^{\infty}(M, N), g \in G$. Let $\mathscr{Q}_{k}^{G}$ denote the $G$-equivariant elements of $\mathscr{D}_{k}$. Full definitions are given in $\S 2$.

There are two equivariance preserving structures on $\mathscr{D}_{\infty}$ each with its own generation problem. The first structure is a multiplication: $\mathscr{D}_{k} \times \mathscr{D}_{k} \rightarrow \mathscr{D}_{k}$, defined by

$$
\mathscr{F}_{1} \cdot \mathscr{F}_{2}(f)=\mathscr{F}_{1}(f) \mathscr{F}_{2}(f), f \in C^{\infty}(M, N) .
$$

If $N=\mathbf{R}$, a second structure is induced by the composition $\mathscr{D}_{k_{1}} \times \mathscr{D}_{k_{2}} \rightarrow$ $\mathscr{O}_{k_{1}+k_{2}}$,given by

$$
\mathscr{F}_{1} \mathscr{F}_{2}(f)=\mathscr{F}_{1}\left(\mathscr{F}_{2}(f)\right), f \in C^{\infty}(M, \mathbf{R}) .
$$

The main results of this paper are two finiteness theorems, one for each of these structures.

THeOReM 1. Let $G$ be a compact Lie group, $M$ a smooth $G$-manifold of finite orbit type and $N$ a smooth manifold then, for each $k \in\{0,1,2, \ldots\}$, there exist $\mathbb{Q}_{1}, \ldots, \mathbb{Q}_{i} \in \mathscr{D}_{k}^{G}$ such that $\mathscr{F} \in \mathscr{D}_{k}^{G}$ iff $\mathscr{F}=f\left(\mathbb{Q}_{1}, \ldots, \mathbb{Q}_{i}\right)$, for some $f \in C^{\infty}\left(\mathbf{R}^{i}\right)$.

This theorem is based on a theorem of Schwarz [10], the proof is given in $\$ 2$.

Let $C^{\infty}(M)^{G}$ denote the $G$-invariant elements of $C^{\infty}(M)$. A function $\xi$ : $M \rightarrow \mathbf{R}^{l}$ is called a finite generator for $C^{\infty}(M)^{G}$ iff $C^{\infty}(M)^{G}=\xi^{*} C^{\infty}(M)$.

Received by the editors December 5, 1977 and, in revised form, February 15, 1978 and October 4, 1978.

AMS (MOS) subject classifications (1970). Primary 57E10, 58G99.

Key words and phrases. Equivariant differential operators, transformation groups, differential invariants.

'The preparation of this paper was supported by the National Research Council under Grant A8731.

(C) 1979 American Mathematical Society $0002-9939 / 79 / 0000-0570 / \$ 03.00$ 
We let $\Gamma^{G}(T(M))$ denote the $C^{\infty}(M)^{G}$-module of invariant vector fields on $M$. In the case where $M$ is a principal $G$-bundle finite generators exist both for $C^{\infty}(M)^{G}$ and for the module $\Gamma^{G}(T(M)$ ) (Lemmas (3.1) and (3.2)). Moreover, $\left\{X_{1}, \ldots, X_{n}\right\} \subset \Gamma^{G}(T(M))$ is a generator for $\Gamma^{G}(T(M))$ iff $\left\{X_{1}(x), \ldots, X_{n}(x)\right\}$ generates the vector space $T_{x}(M)$ for all $x \in M$ (Lemma (3.3)). Let $N=\mathbf{R}$.

THeOREM 2. Let $M$ be a principal $G$-bundle with fibration $\{M, \pi, B\}$, $\xi$ : $M \rightarrow \mathbf{R}^{l}$ a generator for $C^{\infty}(M)^{G}$ and $\left\{X^{1}, \ldots, X^{n}\right\}$ a generator for $\Gamma^{G}(T(M))$. Then

for $k>1$.

$$
\mathscr{D}_{k}(M)^{G}=\left\{\xi,\left(X^{\alpha}\right)_{|\alpha|<k}\right\}^{*} C^{\infty}\left(\mathbf{R}^{l} \times \mathbf{R}^{n^{k}}\right)
$$

$\left(X^{\alpha}\right)_{|\alpha|<k}$ denotes the sequence of all $X^{\alpha}$ with $|\alpha|<k$ in lexicographical order and $\left\{\xi,\left(X^{\alpha}\right)_{|\alpha|<k}\right\}^{*} C^{\infty}\left(\mathbf{R}^{l} \times \mathbf{R}^{n^{k}}\right)$ is the set of operators of the form $a\left(\xi,\left(X^{\alpha}\right)_{|\alpha|<k}\right), a \in C^{\infty}\left(\mathbf{R}^{l} \times \mathbf{R}^{n^{k}}\right)$.

In a somewhat different context, problems of this type were studied by Lie [8], by Tresse [11] and more recently by Kumpera [7].

Thanks are due to Ivan Kupka for some helpful discussion.

2. The verification of Theorem 1. Let $J^{k}(M, N)$ be the $k$ th jet bundle from $M$ into $N$ with source map $\alpha$ and target map $\beta$. If $P$ and $Q$ are smooth manifolds, $\mu: P \rightarrow M$ a diffeomorphism, $\nu: N \rightarrow Q$ a smooth map, then $\left(J^{k} \mu\right)^{*}: J^{k}(M, N) \rightarrow J^{k}(P, N)$ and $\left(J^{k} \nu\right)_{*}: J^{k}(M, N) \rightarrow J^{k}(M, Q)$ are defined by

$$
\left(J^{k} \mu\right)^{*}(\sigma)=j_{\mu^{-1}(\alpha(\sigma))}^{k} f \circ \mu \quad \text { and } \quad\left(J^{k} \nu\right)_{*}(\sigma)=j_{\alpha(\sigma)}^{k} \nu \circ f
$$

where $f$ represents $\sigma$.

The action of $G$ on $M$ lifts to a smooth action on $J^{k}(M, N)$ by

$$
g \cdot \sigma=\left(J^{k} \phi_{g}^{-1}\right) *(\sigma) .
$$

Let $\pi_{k}$ be the canonical projection of $J^{k+1}(M, N)$ onto $J^{k}(M, N)$ and put $D_{k}=C^{\infty}\left(J^{k}(M, N), \mathbf{R}\right) . D_{\infty}$ is the inductive limit as $k \rightarrow \infty$ of $\left(D_{k}, \pi_{k}^{*}\right)$, where $\pi_{k}^{*}$ is the map from $D_{k}$ to $D_{k+1}$ given by $\pi_{k}^{*} F=F \circ \pi_{k}$.

The set of smooth nonlinear differential operators from $C^{\infty}(M, N)$ into $C^{\infty}(M, \mathbf{R})$ of order $<k, k \in\{\infty, 1,2,3, \ldots\}$ is denoted by $\mathscr{D}_{k} . \mathscr{F} \in \mathscr{D}_{k}$ iff it factorizes as $F \circ j^{k}$, for some $F \in D_{k}$. In this case $F$ is called the symbol of $\mathscr{F}$ or $F=\operatorname{sym} \mathscr{F}$. The $G$-equivariant elements of $\mathscr{D}_{k}$ are denoted by $\mathscr{D}_{k}^{G}$, the $G$-invariant elements of $D_{k}$ by $D_{k}^{G}$.

(2.2) Proposition. $\mathscr{F} \in \mathscr{D}_{k}^{G}$ iff sym $\mathscr{F} \in D_{k}^{G}$.

Proof. Let $F$ be the symbol of $\mathcal{F}$. If $F$ is $G$-invariant, $f \in C^{\infty}(M, N)$, $x \in M$, then

$$
\begin{aligned}
\mathscr{F}(g \cdot f)(x) & =F\left(j_{x}^{k} g \cdot f\right)=\left(g^{-1} \cdot F\right)\left(j_{g^{-1} \cdot x}^{k} f\right)=F\left(j_{g^{-1} \cdot x}^{k} f\right) \\
& =(\mathscr{F} f)\left(g^{-1} \cdot x\right)=(g \cdot \mathscr{F} f)(x) .
\end{aligned}
$$


Conversely, if $\mathscr{F}$ is $G$-equivariant and $\sigma \in J^{k}(M, N)$ with $\alpha(\sigma)=x$ is represented by $f$ then

$$
\begin{aligned}
g \cdot F(\sigma) & =F\left(j_{g^{-1} \cdot x}^{k} g^{-1} \cdot f\right)=\mathscr{F}\left(g^{-1} \cdot f\right)\left(g^{-1} \cdot x\right) \\
& =\left(g^{-1} \cdot \mathscr{F} f\right)\left(g^{-1} \cdot x\right)=\mathscr{F} f(x)=F(\sigma) .
\end{aligned}
$$

(2.3) LEMMA. Let $G$ be a compact Lie group and $M$ a smooth $G$-manifold with orbit structure of finite type (see [9]), then the induced action on $J^{k}(M, N)$ is of finite orbit type as well.

Proof. If $N=\mathrm{R}^{n}$ and $M$ is a linear $G$-space then $J^{k}(M, N)$ is a linear $G$-space which is of finite orbit type. In the general case we can assume by the Whitney and Mostov embedding theorems that $N$ is smoothly embedded in $\mathbf{R}^{n}$ and $M$ is smoothly equivariantly embedded in a Euclidean $G$-space $\mathbf{R}^{m}$. It will suffice to show that $J^{k}(M, N)$ is equivariantly embedded in $J^{k}\left(\mathbf{R}^{m}, \mathbf{R}^{n}\right)$. Let $\pi: Z \rightarrow M$ be an equivariant tubular neighbourhood of $M$ in $\mathbf{R}^{m}$. Since $Z$ is an open $G$-invariant set in $\mathbf{R}^{m}, J^{k}\left(Z, \mathbf{R}^{n}\right)$ is an open $G$-submanifold of $J^{k}\left(\mathbf{R}^{m}, \mathbf{R}^{n}\right)$, so we need only show that $J^{k}(M, N)$ is equivariantly embedded in $J^{k}\left(Z, \mathbf{R}^{n}\right)$.

Let $i$ be the inclusion map of $N$ in $\mathbf{R}^{n}$. Clearly, $\left(J^{k} i\right)_{*}: J^{k}(M, N) \rightarrow$ $J^{k}\left(M, \mathbf{R}^{n}\right)$ and $\left(J^{k} \pi\right)^{*}: J^{k}\left(M, \mathbf{R}^{n}\right) \rightarrow J^{k}\left(Z, \mathbf{R}^{n}\right)$ are equivariant embeddings. $\left(J^{k} \pi\right)^{*} \circ\left(J^{k} i\right)_{*}$ is the desired equivariant embedding of $J^{k}(M, N)$ into $J^{k}\left(Z, \mathbf{R}^{n}\right)$.

Thanks are due to the referee of an earlier version of this section for shortening my original proof.

By Lemma (2.3), the conditions of Theorem 1 imply that the $G$-manifold $J^{k}(M, N)$ is of finite orbit type. By a theorem of G. W. Schwarz [10, Theorem 2], there exist $A_{1}, \ldots, A_{i} \in D_{k}^{G}$, such that $F \in D_{k}^{G}$ iff $F=f\left(A_{1}, \ldots, A_{i}\right)$, for some $f \in C^{\infty}\left(\mathbf{R}^{i}, \mathbf{R}\right)$. Hence, the operators $Q_{l}, 1<l<i$, may be chosen as those with $\operatorname{sym} Q_{l}=A_{l}$.

3. The verification of Theorem 2 . In this section, $M$ is a smooth principal $G$-bundle with corresponding fibration $\{M, \pi, B\} . m=\operatorname{dim} M, a=\operatorname{dim} G$ and $b=m-a=\operatorname{dim} B$.

(3.1) LeMMA. There exists an invariant generator $\xi: M \rightarrow \mathbf{R}^{l}, l<2 b+1$, for $C^{\infty}(M)^{G}$.

Proof. By Whitney's embedding theorem there exists an embedding $\zeta$ of $B$ into $\mathbf{R}^{p}, p<2 b+1$. Since $C^{\infty}(M)^{G}=\pi^{*} C^{\infty}(B)$, we may choose $\xi=\zeta \circ \pi$.

The action of $G$ on $M$ lifts in the usual way to $T(M)$ by $g \cdot(x, v)=(g \cdot$ $x, g \cdot v)$, where $x \in M, v \in T_{x}(M), g \cdot x=\phi_{g}(x)$ and $g \cdot v=d \phi_{g}(x) v$. As in $\S 1, \Gamma^{G}(T(M))$ denotes the $C^{\infty}(M)^{G}$-module of $G$-invariant vector fields on M.

(3.2) LemMA. There exists a finite generator for $\Gamma^{G}(T(M))$. 
Proof. For each $x \in M$, let $H_{x}(M)$ be the horizontal tangent space to $M$ at $x$ with respect to a given principal connection $\mathscr{P}$ on $M$ and let $V_{x}(M)$ be the vertical tangent space at $x$. In $T(M)$ we consider the subbundles $H(M)=\cup_{x \in M} H_{x}(M)$ and $V(M)=\cup_{x \in M} V_{x}(M)$. Since $T(M)=H(M)$ $\bigoplus V(M)$, we need only show that finite generators exist for $\Gamma^{G}(H(M))$ and $\Gamma^{G}(V(M))$, the $C^{\infty}(M)^{G}$-modules of $G$-invariant horizontal and vertical vector fields on $M$.

First we construct a finite generator for $\Gamma^{G}(H(M))$. By Whitney's embedding theorem we may assume that $B$ is embedded in $\mathbf{R}^{p}, p<2 b+1$. Projecting the canonical basis of $T\left(\mathbf{R}^{p}\right)$ onto $T(B)$ we obtain a generator for $T(B)$. The horizontal liftings with respect to $\mathcal{P}$ of the elements of this generator constitute a generator for $\Gamma^{G}(H(M))$.

$V(M)$ is a $G$-subbundle of the $G$-vector bundle $T(M)$. The action of $G$ on $V(M)$ is given by

where $(x, v) \in M \times V_{x}(M)$.

$$
g \cdot(x, v)=(g \cdot x, g \cdot v)
$$

Let $\operatorname{Lie}(G)$ be the Lie algebra of $G$. For $l \in \operatorname{Lie}(G)$, let the vertical vector field $\vec{l}$ on $M$ be defined by

$$
\vec{l}(x)=\left.\frac{d}{d t}\left(e^{t l}(x)\right)\right|_{t=0}, \quad x \in M
$$

We define a left $G$-action on $M \times \operatorname{Lie}(G)$ by

$$
g \cdot(x, l)=(g \cdot x, \operatorname{Ad}(g) l)
$$

where $\operatorname{Ad}(g)$ is the adjoint action of $g \in G$ on $\operatorname{Lie}(G)$. With this action, $M \times \operatorname{Lie}(G)$ is isomorphic, as a $G$-vector bundle, to $V(M)$. An isomorphism is given by $\omega: M \times \operatorname{Lie}(G) \rightarrow V(M)$ :

$$
\omega(x, l)=(x, \vec{l}(x))
$$

We check that $\omega$ is $G$-equivariant:

$$
\begin{aligned}
\omega(g \cdot(x, l)) & =\omega(g \cdot x, \operatorname{Ad}(g) l)=(g \cdot x, \overrightarrow{\operatorname{Ad}(g) l}(g \cdot x)) \\
& =(g \cdot x, g \cdot \vec{l}(x))=g \cdot \omega(x, l),
\end{aligned}
$$

for all $g \in G$ and $(x, l) \in M \times \operatorname{Lie}(G)$.

Let $E=M \times{ }^{G} \operatorname{Lie}(G)$ be the vector bundle over $B$ of fiber type $\operatorname{Lie}(G)$ associated with the principal bundle $M$ and the adjoint action of $G$ on $\operatorname{Lie}(G)$ [4, XVI, 16.14.7]. In our case $E$ is the quotient of $M \times \operatorname{Lie}(G)$ by the action defined by (3.5). The invariant vertical vector fields on $M$ are in bijective correspondence to the sections of $E$, (see e.g. [6, Theorem 4.8.1]). Since the $C^{\infty}(B)$-module of cross sections of $E$ is finitely generated, [5, p. 76, Lemma 2], the same is true for the $C^{\infty}(M)^{G}$-module $\Gamma^{G}(V(M))$. This completes the proof.

(3.7) LEMMA. $\left\{X^{1}, \ldots, X^{n}\right\} \subset \Gamma^{G}(T(M))$ is a generator for $\Gamma^{G}(T(M))$ iff $\left\{X^{1}(x), \ldots, X^{n}(x)\right\}$ generates the vector space $T_{x}(M)$, for all $x \in M$. 
Proof. Clearly a generator of $\Gamma^{G}(T(M))$ generates the individual tangent spaces. To prove the converse, let

$$
\left\{X^{1}, \ldots, X^{n}\right\} \subset \Gamma^{G}(T(M))
$$

be such $\left\{X^{1}(x), \ldots, X^{n}(x)\right\}$ generates $T_{x}(M)$, for all $x \in M$. For each $x \in X$ we may choose a subset of (3.8)

$$
\left\{X^{j_{1}(x)}, \ldots, X^{j_{m}(x)}\right\}
$$

which, evaluated at $x$, is a basis for $T_{x}(M)$. Being a basis is an open condition so there exists an open neighbourhood $0_{x}$ of $x$ such that (3.9), evaluated at any $y \in 0_{x}$ is a basis for $T_{y}(M)$. Because $g \cdot X^{i}(x)=X^{i}(g \cdot x), x \in M$ and $1<i<n$, and since the action of $G$ on $T(M)$ preserves linear independence (3.9), evaluated at $y$ is a basis for $T_{y}(M)$ for all $y \in G \cdot 0_{x}=\pi^{-1}\left(U_{x}\right)$, where $U_{x}=\pi\left(0_{x}\right)$.

Let $\left\{V_{\alpha}\right\}_{\alpha \in I}$ be a locally finite refinement of the covering $\left\{U_{x}\right\}_{x \in M}$ of $B$. By the above construction, for each $\alpha \in I$, we have a subset of $m$ elements of (3.8).

$$
\left\{X^{j_{\alpha}(1)}, \ldots, X^{j_{\alpha}(m)}\right\}
$$

which, evaluated at any $x \in \pi^{-1}\left(V_{\alpha}\right)$, is a basis for $T_{x}(M)$. Hence any $Y \in \Gamma^{G}(T(M))$ is of the form

$$
Y(x)=\sum_{i=1}^{m} a_{j_{\alpha}(i)}(x) X^{j_{\alpha}(i)}(x), \quad x \in \pi^{-1}\left(V_{\alpha}\right),
$$

where $a_{j_{\alpha}(i)} \in C^{\infty}\left(\pi^{-1}\left(V_{\alpha}\right)\right)^{G}, 1 \leqslant i \leqslant m, \alpha \in I$.

Let $\left\{f_{\alpha}\right\}_{\alpha \in I}$ be a partition of unity subordinate to $\left\{V_{\alpha}\right\}_{\alpha \in I}$ with $\operatorname{supp} f_{\alpha} \subset$ $V_{\alpha}, \forall \alpha \in I$. Then

$$
Y=\sum_{\alpha \in I} f_{\alpha} \circ \pi \sum_{i=1}^{m} a_{j_{\alpha}(i)} X^{j_{\alpha}(i)}
$$

which may be written as

$$
Y=\sum_{i=1}^{n} b_{i} X^{i}
$$

with $b_{i} \in C^{\infty}(M)^{G}, 1 \leqslant i<m$, since each $b_{i}$ is locally a finite sum of functions $\left(f_{\alpha} \circ \pi\right) a_{j_{\alpha}(i)}$ which are smooth and $G$-invariant.

Proof of Theorem 2. First we consider the case where $M$ is a trivial principal bundle: $M=V \times G$. Moreover we assume that $V$ is an open set of $\mathbf{R}^{b}$. For $k \in\{1,2,3, \ldots\}$ let $A_{k}: J^{k}(V \times G) \rightarrow J_{V \times\{e\}}^{k}(V \times G)$ be defined by

$$
A_{k}(\sigma)=g^{-1} \cdot \sigma \quad \text { if } \alpha(\sigma)=(v, g)
$$

Then $\left\{J^{k}(V \times G), A_{k}, J_{V \times\{e\}}^{k}(V \times G)\right\}$ is a fibration of the principal $G$-bundle $J^{k}(V \times G)$, so

$$
C^{\infty}\left(J^{k}(V \times G)\right)^{G}=A_{k}^{*} C^{\infty}\left(J_{V \times\{e\}}^{k}(V \times G)\right) .
$$


The mapping $B_{k}$, defined by

$$
B_{k}\left(j_{(b, e)}^{k} f\right)=\left(b, j_{(0, e)}^{k}\left(f \circ t_{b}\right)\right),
$$

where $f \in C^{\infty}\left(\mathbf{R}^{b} \times G\right)$ and $t_{b}$ is the translation in $\mathbf{R}^{b} \times G$, given by $t_{b}(a, h)$ $=(a+b, h)$, with $b \in \mathbf{R}^{b}$ and $(a, h) \in \mathbf{R}^{b} \times G$, is a diffeomorphism of $J_{\mathbf{R}^{b} \times\{e\}}^{k}\left(\mathbf{R}^{b} \times G\right)$ onto $\mathbf{R}^{b} \times J_{(0, e)}^{k}\left(\mathbf{R}^{b} \times G\right)$. The space $J_{(0, e)}^{k}\left(\mathbf{R}^{b} \times G\right)$ carries a natural linear structure. By choosing a basis it is identified with $\mathbf{R}^{N}, N=$ $\operatorname{dim} J_{(0, e)}^{k}\left(R^{b} \times G\right)$ and we may consider $B_{k}$ as a diffeomorphism of $J_{\mathbf{R}^{b} \times\{e\}}^{k}\left(\mathbf{R}^{b} \times G\right)$ onto $\mathbf{R}^{b} \times \mathbf{R}^{N}$. From (3.13) we obtain

$$
C^{\infty}\left(J^{k}(V \times G)\right)^{G}=\left(B_{k} \circ A_{k}\right)^{*} C^{\infty}\left(V \times \mathbf{R}^{N}\right) .
$$

The canonical projections of $\mathbf{R}^{b} \times \mathbf{R}^{N}$ on its first and second factors are denoted by $\mathrm{pr}_{1}$ and $\mathrm{pr}_{2}$. Let $\mathscr{P}_{k}: C^{\infty}(V \times G) \rightarrow C^{\infty}\left(V \times G, \mathbf{R}^{N}\right)$ be the $G$-equivariant linear operator defined by

$$
\mathscr{P}_{k}=\mathrm{pr}_{2} \circ B_{k} \circ A_{k} \circ j^{k}
$$

and let $\pi^{\prime}$ be the canonical projection of $V \times G$ onto $V$. From Proposition (2.2) and formula (3.15) it follows that $\mathbb{Q} \in \mathscr{D}_{k}^{G}(V \times G)$ iff there exists some $a \in C^{\infty}\left(\mathbf{R}^{b} \times \mathbf{R}^{N}\right)$ such that, for all $f \in C^{\infty}(V \times G)$,

$$
\text { Qf }=a\left(\mathrm{pr}_{1} \circ B_{k} \circ A_{k} \circ j^{k}, \mathrm{pr}_{2} \circ B_{k} \circ A_{k} \circ j^{k}\right)
$$

which equals a $\left(\pi^{\prime}, \mathscr{P}_{k} f\right)=a\left(\pi^{\prime}, \mathscr{P}_{k}\right) f$. Hence

$$
\mathscr{D}_{k}^{G}(V \times G)=\left(\pi^{\prime}, \mathscr{P}_{k}\right)^{*} C^{\infty}\left(\mathbf{R}^{b} \times \mathbf{R}^{N}\right) \text {. }
$$

Let $\mathscr{P}_{k}^{i}, 1<i<N$, denote the $i$ th component of $\mathscr{P}_{k}$. Clearly each $\mathscr{P}_{k}^{i}$ is a linear $G$-equivariant differential operator on $C^{\infty}(V \times G)$.

It is easy to see that there exists an invariant basis

$$
\left\{Y^{1}, \ldots, Y^{m}\right\}
$$

for $T(V \times G)(m=\operatorname{dim} V \times G)$. From the linearity of $\mathscr{P}_{k}$ it follows that each $\mathscr{P}_{k}^{i}, 1<i<N$, can be written uniquely as

$$
\mathscr{P}_{k}^{i}=\sum_{|\alpha|<k} a_{i}^{\alpha} Y^{\alpha}, \quad a_{i}^{\alpha} \in C^{\infty}(V \times G),
$$

(see e.g. [12, Theorem 1.1.2]). It follows from the $G$-equivariance of $\mathscr{P}_{k}^{i}$ and of the operators $Y^{\alpha},|\alpha| \leqslant k$, that the coefficients $a_{i}^{\alpha},|\alpha|<k, 1<i<N$, are $G$-invariant. Thus we get the $\mathscr{P}_{k}^{i}$ in the form

$$
\mathscr{P}_{k}^{i}=\sum_{|\alpha|<k}\left(b_{i}^{\alpha} \circ \pi^{\prime}\right) Y^{\alpha}, \quad 1 \leqslant i \leqslant N,
$$

where $b_{i}^{\alpha} \in C^{\infty}(V),|\alpha|<k, 1<i<N$.

Substituting (3.21) into (3.18) we obtain that $\mathscr{Q} \in \mathscr{D}_{k}^{G}(V \times G)$ iff there exists some $a \in C^{\infty}\left(\mathbf{R}^{b} \times \mathbf{R}^{N}\right)$ such that

$$
\mathbb{Q} f=a\left(\pi^{\prime}, \sum_{|\alpha|<k}\left(b_{1}^{\alpha} \circ \pi^{\prime}\right) Y^{\alpha} f, \ldots, \sum_{|\alpha|<k}\left(b_{N}^{\alpha} \circ \pi^{\prime}\right) Y^{\alpha} f\right),
$$

for all $f \in C^{\infty}(V \times G)$. The right-hand side of (3.22) is just a function of $\pi^{\prime}$ 
and the operators $Y^{\alpha},|\alpha| \leqslant k$. Conversely, any such function represents an element of $\mathscr{D}_{k}^{G}(V \times G)$. Hence,

$$
\mathscr{D}_{k}^{G}(V \times G)=\left(\pi^{\prime},\left(Y^{\alpha}\right)_{|\alpha|<k}\right) C^{\infty}\left(\mathbf{R}^{b} \times \mathbf{R}^{m^{k}}\right) .
$$

Let $\left\{U_{e}\right\}_{e \in I}$, where $I$ is some index set, be a locally finite atlas for $B$ such that the principal bundles $M_{e}$ induced by $M$ over $U_{e}, \varepsilon \in I$, are trivializable. Then for each $\varepsilon \in I$ there exists an isomorphism $\lambda_{\varepsilon}$ of $M_{\varepsilon}$ onto the product bundle $V_{e} \times G$, where $V_{\varepsilon} \subset \mathbf{R}^{b}$ is the parameter domain of $U_{\varepsilon}$.

We define a bijection $\Lambda_{\varepsilon}^{k}$ of $\mathscr{D}_{\infty}^{G}\left(V_{\varepsilon} \times G\right)$ onto $\mathscr{D}_{\infty}^{G}\left(M_{\varepsilon}\right)$ by

$$
\left(\Lambda_{e} \mathscr{Q}\right) f=\lambda_{\varepsilon}^{*}\left(\mathscr{Q}\left(f \circ \lambda_{\varepsilon}^{-1}\right)\right), \quad f \in C^{\infty}\left(M_{e}\right) .
$$

Let $\pi_{e}^{\prime}$ be the canonical projection of $V_{\varepsilon} \times G$ onto $V_{\varepsilon}$ and let $\left\{Y_{e}^{1}, \ldots, Y_{e}^{m}\right\}$ be a $G$-invariant basis for $T\left(V_{e} \times G\right)$. From (3.23) and (3.24) we obtain that $\mathscr{B} \in \mathscr{D}_{k}^{G}\left(M_{e}\right)$ iff

$$
\mathscr{B}=\Lambda_{e}\left(q\left(\pi_{e}^{\prime},\left(Y_{e}^{\alpha}\right)_{|\alpha|<k}\right)\right),
$$

for some $q \in C^{\infty}\left(\mathbf{R}^{l} \times \mathbf{R}^{m^{k}}\right)$. Or

$$
\mathscr{B}=q\left(\pi_{e}^{\prime} \circ \lambda_{\varepsilon},\left(\left(\Lambda_{\varepsilon} Y_{\varepsilon}\right)^{\alpha}\right)_{|\alpha|<k}\right) .
$$

Let $\xi_{\varepsilon}: M_{\varepsilon} \rightarrow \mathbf{R}^{l}$ and $\left\{X_{\varepsilon}^{1}, \ldots, X_{\varepsilon}^{n}\right\}$ be the restrictions of the given generators for $C^{\infty}(M)^{G}$ and $\Gamma^{G}(T(M))$ to $M_{\varepsilon}$. Then

$$
\pi_{e}^{\prime} \circ \lambda_{\varepsilon}=d \circ \xi_{e}
$$

for some $d \in C^{\infty}\left(\mathbf{R}^{l}, \mathbf{R}^{b}\right)$. Since $\left\{X_{e}^{j}(x), \ldots, X_{e}^{j}(x)\right\}$ generates $T_{x}\left(M_{e}\right), \forall x \in$ $M_{e}$, it follows from Lemma (3.7) that

$$
\Lambda_{e} Y_{e}^{i}=\sum_{j=1}^{n}\left(e_{e}^{i j} \circ \xi_{e}\right) X_{e}^{j},
$$

where $e_{e}^{i j} \in C^{\infty}\left(\mathbf{R}^{l}\right), 1<i<m, 1<j<n$. After substitution of (3.27) and (3.28) into (3.26) it is easy to see that we may write this equality as

$$
\mathscr{B}=r\left(\xi_{e},\left(X_{e}^{\alpha}\right)_{|\alpha|<k}\right),
$$

for some $r \in C^{\infty}\left(\mathbf{R}^{l}, \mathbf{R}^{n^{k}}\right)$.

Let $\left\{u_{e}\right\}_{e \in I}$ be a partition of unity on $\mathscr{B}$ subordinate to $\left\{U_{e}\right\}_{e \in I}$ with $\operatorname{supp}\left(u_{e}\right) \subset U_{e}, \varepsilon \in I$. For a given $\mathscr{F} \in \mathscr{D}_{k}^{G}(M)$ let $\mathscr{F}_{\varepsilon} \in \mathscr{D}_{k}^{G}\left(M_{e}\right)$ be the restriction of $\mathscr{F}$ to $C^{\infty}\left(M_{e}\right) .\left(F_{e}\right.$ is defined by $\left.\operatorname{sym} \mathscr{F}_{e}=\left.\operatorname{sym} \mathscr{F}\right|_{J^{k}\left(M_{e}\right)}\right)$ By (3.29) there exists a $r_{e} \in C^{\infty}\left(\mathbf{R}^{l} \times \mathbf{R}^{n^{k}}\right)$ such that

$$
\mathscr{F}_{\varepsilon}=r_{\varepsilon}\left(\xi_{e},\left(X_{\varepsilon}^{\alpha}\right)_{|\alpha|<k}\right)
$$

So

$$
\mathscr{F}=\sum_{\varepsilon \in I}\left(u_{\varepsilon} \circ \pi\right) r_{\varepsilon}\left(\xi_{e},\left(X_{\varepsilon}^{\alpha}\right)_{|\alpha|<k}\right)
$$

Since

$$
\left(U_{e} \circ \pi\right) r_{e}\left(\xi_{e},\left(X_{e}^{\alpha}\right)_{|\alpha|<k}\right)=\left(U_{e} \circ \pi\right) r_{e}\left(\xi,\left(X^{\alpha}\right)_{|\alpha|<k}\right)
$$

it follows from (3.31) that

$$
\mathscr{F}=a\left(\xi,\left(X^{\alpha}\right)_{|\alpha|<k}\right),
$$


for some $a \in C^{\infty}\left(\mathbf{R}^{l} \times \mathbf{R}^{n^{k}}\right)$. Conversely, (3.32) implies that $\mathscr{F} \in \mathscr{D}_{k}^{G}(M)$. This completes the proof.

\section{BIBLIOGRAPHY}

1. S. Bochner and D. Montgomery, Groups of differentiable and real or complex analytic transformations, Ann. of Math. 46 (1945), 685-694.

2. N. Bourbaki, Lie groups and Lie algebras, Part I, Addison-Wesley, Reading, Mass., 1975.

3. R. Delver, Equivariant differential operators of a Lie group. Transformation groups, (Proc. Conf., Univ. Newcastle-upon-Tyne, August 1976), edited by C. Kosniowski, Cambridge Univ. Press, London, 1977.

4. J. Dieudonné, Treatise on analysis, Vol. III, Pure and Appl. Math., Academic Press, New York, 1972.

5. W. Greub, S. Halperin and R. Vanstone, Connections, curvature and cohomology, Vol. 1, Academic Press, New York, 1972.

6. D. Husemoller, Fibre bundles, 2nd ed., Graduate Texts in Math., Springer-Verlag, Berlin, 1975.

7. A. Kumpera, Invariants différentiels d'un pseudogroupe de Lie. I, J. Differential Geometry 10 (1975), 289-345; II, ibid. 10 (1975), no. 3, 347-416.

8. S. Lie, Allgemeine Untersuchungen über Differentialgleichungen die eine kontimuierliche, endliche Gruppe gestatten, Math. Ann. 25 (1885), 77-151; Gesammete Abhandlungen, Bd. VI, Teubner, Leipzig, 1927, 139-223.

9. R. S. Palais, Slices and equivariant imbeddings, Seminar in Transformation Groups, Chapter VIII, Ann. of Math. Studies, no. 46, Princeton Univ. Press, Princeton, N.J., 1968.

10. G. W. Schwar, Smooth functions invariant under the action of a compact Lie group, Topology 14 (1975), 63-68.

11. A. Tresse, Sur les invariants differentiels des groupes continus de transformations, Thèse, Paris, 1893; Acta Math. 18 (1894), 1-88.

12. V. S. Varadarajan, Lie groups, Lie algebras, and their representations, Prentice-Hall, Englewood Cliffs, N.J., 1974.

Department of Mathematics, University of Toronto, Toronto, Ontario, Canada M5S-1A1

Current address: Department of Mathematics, Free University, 1007 MC, Amsterdam, The Netherlands 